Revista Calidad en la Educación Superior

Programa de Autoevaluación Académica

Universidad Estatal a Distancia

ISSN 1659-4703

Costa Rica

revistacalidad@uned.ac.cr

Abordaje de factores de éxito académico desde la unidad académica:

El caso de la escuela Ciencias Geográficas,

Universidad Nacional, Costa Rica

Addressing academic success factors from the academic unit:

Geographical Sciences School's case

National Universidad, Costa Rica

Lic. Iliana Araya Ramírez

Académica de la Escuela de Ciencias Geográficas de la Universidad Nacional (ECG-UNA). Coordinadora de la Comisión de Autoevaluación de la Carrera de Ciencias Geográficas

MSc. Lilliam Quirós Arias

Académica de la Escuela de Ciencias Geográficas de la Universidad Nacional. Subdirectora de la ECG-UNA

Lic. Amalia Ruiz Hernández

Académica de la Escuela de Ciencias Geográficas de la Universidad Nacional. Miembro comisión de Autoevaluación de la ECG-UNA.

Volumen 1, Número 1

Mayo 2010

pp. 1 - 19

Recibido: abril, 2010

Aprobado: mayo, 2010 


\section{Abordaje de factores de éxito académico desde la unidad académica: El caso de la escuela Ciencias Geográficas, Universidad Nacional, Costa Rica.}

\section{Resumen}

La educación superior enfrenta el reto de responder al cambio social, tecnológico y a las nuevas formas de organización del trabajo. La autoevaluación de carreras universitarias contribuye al mejoramiento continuo de las prácticas de gestión curricular y de la calidad de la educación impartida. La carrera de Ciencias Geográficas con énfasis en Ordenamiento del Territorio de la Universidad Nacional (CCG-UNA) inició un proceso de autoevaluación con miras a la acreditación desde el año 2005, éste ha permitido visibilizar algunos de los factores que contribuyen al éxito académico.

El propósito de esta ponencia es identificar los factores de éxito académico que inciden en la graduación, inserción al mercado de trabajo, deserción y rezago.

Palabras clave: educación superior, autoevaluación, gestión curricular y de la calidad, éxito académico.

\section{Summary}

Higher education is challenged to respond to social changing, technological and new forms of work organization. The university self-assessment contributes to the continuous improvement of management practices curriculum and the quality of education provided. The college degree of Geographical Sciences, with emphasis on Land Use Planning National University (CCG-UNA) initiated a process of selfassessment with a view to accreditation since 2005 , it has enabled more visible some of the factors that contribute to academic success. The purpose of this paper is to identify factors that influence academic success at graduation to the labor market integration, desertion and lag.

Keywords: higher education, self-assessment, curriculum management and quality, academic success. 
La estrategia metodológica consistió en el análisis estadístico descriptivo de las cohortes para los años 1999-2009 y se generaron los indicadores de graduación, deserción y rezago. También se realizaron cuestionarios y talleres con los sujetos de investigación. A partir de esta información se formuló una estrategia conducente al éxito académico plasmada en acciones como: seguimiento de graduados, implementación de la guía académica, seguimiento a estudiantes desertores y rezagados.

Desde el año 2005 se inició el seguimiento a graduados, para esto se completó una base de datos que contiene referencias personales, año de graduación, lugar de trabajo, nombre del empleador y funciones desempeñadas. Con la información generada se construyeron indicadores de inserción laboral de los graduados: tasa de ocupación, jornada laboral, tareas realizadas, grado de relación de las tareas con la formación recibida, satisfacción del trabajo desempeñado, empresas donde laboran y demanda de la carrera. Estos indicadores son utilizados en la toma de decisiones y las revisiones curriculares del plan de estudio en vigencia.

A partir del año 2007 y con base en la normativa institucional, la ECG-UNA puso en práctica la asesoría académica, ésta se inició con los estudiantes de primer nivel y para el año 2009 la totalidad de la población estudiantil contó con guía académica. La asesoría se ha realizado de manera grupal e individual, con reuniones de retroalimentación en las cuales participan la mayoría de los docentes. Los estudiantes atendidos manifiestan que la guía académica es una fortaleza para avanzar en el plan de estudio.

El análisis de la deserción permitió la caracterización socioeconómica de la población desertora y la identificación de las causas. A partir de este diagnóstico es posible afinar estrategias de intervención que fortalezcan la permanencia de los estudiantes en la carrera y en la educación superior. La identificación de los estudiantes rezagados facilitó el seguimiento individual y la toma de acciones como remisión a los cursos ofrecidos por el Programa de Éxito Académico de la UNA.

Los factores que contribuyen al éxito académico de los estudiantes de la CCGUNA están mediados por la disponibilidad y compromiso de los docentes de acompañar al estudiante en su proceso formativo y de manera integral. La disponibilidad de información y datos fiables permiten orientar las estrategias de 
intervención conducentes a favorecer la permanencia, la graduación e inserción laboral de los estudiantes que ingresan a la carrera.

\section{Contextualización educación superior y acreditación en Costa Rica}

Costa Rica cuenta con cinco centros públicos de educación superior universitaria: la Universidad de Costa Rica (1940), el Instituto Tecnológico de Costa Rica (1971), la Universidad Nacional (1973), la Universidad Estatal a Distancia (1977) y la Universidad Técnica Nacional (2008). Según datos del 2007, la población de Costa Rica es de 4 476614, la matrícula de la educación superior universitaria es de $1.7 \%$ respecto de la población total. La población de 18 a 22 años es de 446584 (10\% de la población total), de esta un $16.6 \%$ está matriculada en la educación superior universitaria estatal, tendencia que se ha mantenido desde el año 2000 (Rodríguez, 2006. Citado por Quirós, 2009:211).

El 4 de diciembre de 1974, por medio del Convenio de Coordinación de la Educación Superior de Costa Rica, se crearon los organismos que coordinan la educación superior: el Consejo Nacional de Rectores (CONARE), integrado por los rectores de las universidades públicas y la Oficina de Planificación de la Educación Superior (OPES). Además de estos centros de educación pública, han surgido en el país una gran cantidad de centros educativos privados de educación superior, el Consejo Nacional de la Educación Privada (CONESUP), registra 50 centros privados.

El Sistema Nacional de Acreditación de la Educación Superior (SINAES) fue establecido en virtud del acuerdo general suscrito en el convenio para la creación del Sistema Nacional de Acreditación de la Educación Superior aprobado por el Consejo Nacional de Rectores y que entró en vigencia en julio de 1999 al incorporarse a estas cuatro universidades privadas. Posteriormente, la Asamblea Legislativa aprobó la Ley No. 8256, promulgada por el presidente de la república y el Ministro de Educación en el mes de mayo del 2002, mediante la cual se confiere formalmente al SINAES el carácter de organismo nacional oficial de acreditación de la educación superior universitaria costarricense.

Mediante la Gaceta No.94, publicada el 17 de mayo del 2002 se decreta la Ley del Sistema Nacional de la Educación Superior, (SINAES), cuyas actividades se declaran de interés público. Asimismo tendrá como fines planificar, organizar, desarrollar, implementar, controlar y dar seguimiento a un proceso de acreditación que garantice 
continuamente la calidad de las carreras, los planes y programas ofrecidos por las instituciones de educación superior, y salvaguardar la confidencialidad del manejo de los datos de cada institución'.

Por tanto, la acreditación es el proceso de evaluación basado en estándares y criterios de calidad previamente establecidos que es llevado a cabo por un organismo externo y que procura garantizar la calidad de una carrera o programa educativo.

La Universidad Nacional, consciente de la importancia de valorar los parámetros de calidad de sus carreras, inicia el proceso de acreditación en el ano 2001 al momento se tienen acreditadas 10 carreras.

\section{Contextualización de los estudios de deserción y prolongación de estudios en Costa Rica}

Los estudios referentes a la educación superior universitaria estatal en Costa Rica se basan en el seguimiento de cohortes. Tales estudios han sido realizados por la Universidad Nacional, la Universidad de Costa Rica, OPES y CONARE con las cohortes de los años 1990, del 1993 al 1997. Las investigaciones evidencian que un alto porcentaje de los estudiantes admitidos abandona sus estudios sin haber obtenido un grado académico y que gran parte de esta deserción ocurre durante el primer año de estancia en la universidad.

Las investigaciones se han limitado a cuantificar la magnitud del rezago y la deserción de una universidad, sin ahondar en sus causas, ni identificar si se trata sólo de deserción institucional o deserción del sistema. Hasta hace poco tiempo se ha empezado a investigar sobre los factores que inciden en este fenómeno.

\section{Factores que inciden en la deserción y prolongación de los estudiantes de la educación superior universitaria}

La investigación sobre el abandono y la prolongación de los estudios universitarios es un tema que toma cada vez mayor importancia por las repercusiones en el aumento registrado en sus indicadores que se manejan a nivel de la educación superior, las repercusiones se manifiestan según (Brenes, 2005) a nivel institucional, personal y social.

Revista CAES Vol. 1, No. 1, Año 2010

ISSN-1659-4703 
El abandono de la carrera sin finalizarla por parte del alumnado se define como deserción (Cabrera et al, 2006). El rezago es definido como el número de años adicionales, a lo prescrito en el plan de estudio, que un estudiante se mantiene matriculado en una carrera.

Según las investigaciones realizadas la deserción y la prolongación universitaria responden a una situación multicausal y están centradas en el alumnado, el profesorado y la institución (Cabrera et al, 2006). Para Tinto (1975, 1993. Citado por Cabrera et al, 2006), aunque cada estudiante que accede a los estudios superiores viene con sus propios intereses, expectativas e intenciones, lo que determina básicamente su decisión de cambiar o de continuar su proceso formativo es el nivel de integración social y académica que logre en la institución universitaria. Además, la falta de una base formativa que le permita ajustarse a las demandas que plantea el sistema, conllevan al fracaso académico, manifiesto en cambio de carrera, prolongación o abandono de la misma.

Para Bean (1990), la integración social y académica depende de las conductas de afrontamiento positivo (acercamiento) o de afrontamiento negativo (evitación), que inciden directamente en la intención (actitud) de dejar o de continuar los estudios, con lo que se delegan todas las responsabilidades hacia el alumnado (Citado por Cabrera et al, 2006).

Junto a éste, otros factores del alumnado identificados, tanto de naturaleza psicológica como educativa, han sido la falta de capacidades o habilidades para hacer frente a las demandas de los estudios universitarios, conocimientos previos no adecuados, actitudes inapropiadas hacia el aprendizaje, la no correspondencia entre las expectativas del alumnado y las características de la titulación elegida, inadaptación a la estructura y organización académica, estilos de aprendizaje no acordes con la titulación cursada (Cabrera et al, 2006).

En relación con los factores del profesorado cabe destacar las deficiencias pedagógicas (poca claridad en la presentación de la materia; no promover la reflexión ni los juicios críticos; escaso dominio de la materia enseñada; carencia de vocación docente, la falta de atención individualizada al estudiantado y escasa dedicación hacia sus tareas profesionales, como han puesto de manifiesto los estudiantes encuestados por Escandell, Marrero y Castro (2002) de la Universidad de las Palmas de Gran Revista CAES Vol. 1, No. 1, Año 2010 ISSN-1659-4703 
Canaria, o el realizado por Van Ours y Ridder (2003) en Holanda (Citado por Cabrera et al, 2006 ).

La falta de organización académica está relacionada con ausencia de objetivos claramente definidos por parte de la institución (Universidad, Centros, Departamentos), falta de coordinación tanto horizontal como vertical entre el profesorado de una misma titulación y el sistema de acceso a los estudios, restricción en la oferta de determinadas carreras, etc. (Cabrera et al, 2006) desarrolla una investigación con un estudio de casos en una universidad inglesa, y concluye que tanto en el acceso a la universidad como en la finalización de los estudios tiene mucha responsabilidad la interacción entre la institución y otros factores externos de carácter social, como grupo social al que se pertenece, apoyos sociales acordes con las diferentes necesidades.

Levy-Garboua (1986 Citado por Cabrera et al, 2006) propone un modelo economicista con el que intenta explicar que el éxito o el fracaso en el proceso de formación universitaria depende de dos factores: el esfuerzo y las capacidades iniciales del candidato por un lado, y los recursos humanos y materiales que pone a disposición la institución por otro.

Asimismo, el rendimiento académico en la universidad se debería abordar en tres dimensiones: éxito, retraso y abandono. La deserción solamente da cuenta del efecto pero no aporta elementos sobre de las acciones que lo produjeron. La dimensión educacional abordada en el estudio de ambos enfrenta al estudiante con el cambio de concepto.

Todos estos datos nos muestran cómo el abandono de los estudios universitarios es un fenómeno de naturaleza multicausal, Cualquiera que sea el origen, lo que sí es evidente es que las elevadas tasas de abandono o la prolongación del tiempo establecido para concluir la carrera constituyen actualmente uno de los graves problemas al que se debe buscar solución si se pretende mejorar la calidad de la enseñanza superior.

\section{Deserción y prolongación de los estudios universitarios en Costa Rica}

Como señala (Brenes, 2005), los estudios sobre la temática han sido Revista CAES Vol. 1, No. 1, Año 2010

ISSN-1659-4703 
realizados por la OPES, considerando las cohortes de estudiantes 1990 y 1996. Las universidades han realizado estudios en forma independiente, los realizados por la Universidad Nacional y la Universidad de Costa Rica en 1995 y 1999. La Universidad de Costa Rica llevó a cabo otro estudio con las cohortes de 1993 a 1997. La Universidad Estatal a Distancia concluyó la primera parte de un estudio con la cohorte del año 2000.

Estos trabajos dimensionan la problemática de los estudiantes que ingresan y abandonan sus estudios sin obtener un título universitario, además muchos de ellos abandonan las aulas en el primer año de carrera. Sin embargo, los estudios de deserción, han contribuido a la cuantificación de la problemática, hasta recientemente la Universidad de Costa Rica aporta información que permita identificar factores que contribuyen a la deserción.

La deserción y la repitencia conducen a una baja eficiencia de la titulación en las universidades estatales (Brenes, 2005) señala que poco menos de la mitad de los estudiantes que ingresan al sistema obtienen un grado académico y solamente un $10 \%$ lo hacen en el tiempo establecido por el plan de estudio.

Las relaciones encontradas indican que la deserción es mayor cuando: la edad de ingreso a la Universidad es de más de 24 años, el estudiante es casado, proviene de colegios nocturnos, técnicos y oficiales, no ingresó a la carrera deseada, ha realizado estudios universitarios en otra Universidad, ha obtenido un grado académico universitario, trabaja, su núcleo familiar no está compuesto por muchas personas, los poseen tienen bajo nivel educativo, y la principal fuente de financiamiento de los estudios no era una beca (Brenes, 2005).

Para el caso de Costa Rica, el cuadro 1 resume los factores relevantes de la deserción universitaria (Brenes, 2005).

Cuadro 1. Factores relevantes relacionados con la deserción universitaria

\begin{tabular}{|l|l|c|}
\hline \multicolumn{1}{|c|}{$\begin{array}{c}\text { Factores de } \\
\text { deserción }\end{array}$} & \multicolumn{1}{|c|}{ Motivaciones para retirarse } & $\begin{array}{c}\text { Porcentaj } \\
\text { e (\%) }\end{array}$ \\
\hline $\begin{array}{l}\text { Aspectos } \\
\text { institucionales y }\end{array}$ & $\begin{array}{l}\text { Metodología de estudio, larga duración de las } \\
\text { carrera, ambiente de estudio, el personal docente, }\end{array}$ & 25 \\
\hline
\end{tabular}




\begin{tabular}{|l|l|c|}
\hline pedagógicos & la oferta de cursos y la organización administrativa & \\
\hline Aspectos laborales & $\begin{array}{l}\text { Dedicarse a trabajar, para manutención de sus } \\
\text { familia }\end{array}$ & 20 \\
\hline $\begin{array}{l}\text { Insatisfacción con la } \\
\text { carrera }\end{array}$ & $\begin{array}{l}\text { No se siente satisfecho en la carrera que se ubico, } \\
\text { o no haber ingresado a la carrera que deseaba }\end{array}$ & 20 \\
\hline Motivos personales & $\begin{array}{l}\text { Matrimonio, embarazo, problemas emocionales, } \\
\text { salud }\end{array}$ & 15 \\
\hline $\begin{array}{l}\text { Uso parcial de la } \\
\text { institución }\end{array}$ & $\begin{array}{l}\text { Ingresan a dos instituciones para decidirse } \\
\text { posteriormente }\end{array}$ & 10 \\
\hline $\begin{array}{l}\text { Carencia de } \\
\text { financiamiento para } \\
\text { los estudios }\end{array}$ & No cuentan con recursos para cubrir los estudios \\
\hline
\end{tabular}

Fuente: Adaptado Brenes, M. 2005.

Abarca y Sánchez (2005), en un estudio realizado a estudiantes que desertaron en la Universidad de Costa Rica señalan la importancia de diferenciar el tipo de deserción de que se trate. La clasifican como intra-semestral y deserción inter-semestral, tomando como referencia el espacio temporal, esta puede ser parcial o total, además analizan si es deserción institucional o deserción del sistema de educación superior. Para su estudio, el estudiante que abandona las aulas universitarias por cuatro semestres consecutivos se considera un desertor.

Las variables que este mismo estudio toma en cuenta como factores de deserción están relacionados más con situaciones externas al estudiantado, refiriéndose a Otero, s.f., pp.8-9, señalan factores psicosociales y sociodemográficos del contexto familiar, recursos económicos, alguna limitación física y mental, la ausencia de disciplina y el método de estudio. Además de estos factores adicionan como causas de la deserción las expectativas sobre la carrera matriculada, la matrícula en carreras no deseadas, la carga académica, lugar de residencia, oportunidades académicas, la falta de orientación vocacional (Abarca y Sánchez, 2005:7).

Revista CAES Vol. 1, No. 1, Año 2010

ISSN-1659-4703 
Los resultados obtenidos mediante este estudio realizado revelan que el no ingresar a la opción de carrera seleccionada es la principal causa de la deserción. Por lo cual los estudiantes para no quedar fuera del sistema educativo ingresan a otra carrera, en algunos casos, con la expectativa de hacer cambio en el tiempo oportuno, sin embargo, muy pocos (1 de cada 4) lo logran, esto los desmotiva y finalmente desertan ya sea de la educación superior. Un resultado necesario de retomar según este estudio es que un 90,00\% continúan sus estudios en otra Universidad. La falta de información adecuada, antes de una selección de carrera es un elemento importante detectado en este estudio, aspecto que se puede trabajar desde la universidad y unidades académicas.

El abordaje de la deserción realizado por la Universidad Nacional (2008) no dista de los resultados obtenidos en los estudios realizados en Costa Rica. Según la información proporcionada por el estudio, los estudiantes que desertaron manifestaron como razones: no le gustó la carrera, falta de recursos económicos, horarios en los que se ofrecen los cursos y distancia o lejanía de la UNA (Sandoval et al, 2008:76). El 79\% de los estudiantes desertores continúan sus estudios en otra Universidad u otra institución de educación ya sea pública o privada. El escoger la carrera deseada prevalece como un criterio para ingresar a las universidades privadas y públicas. En este sentido, el problema de la deserción es un asunto relevante que impacta la vida de las personas e instituciones, la calidad de los niveles del sistema educativo previos al ingreso a la Universidad, así como la empleabilidad. Su abordaje se requiere de mayor investigación y la identificación de los factores asociados a la deserción y permanencia en la educación superior.

Para hacer frente al problema de la deserción y el rezago se ha propuesto un mayor esfuerzo por lograr: optimización en los procesos de selección e ingreso a carrera, capacitación del personal docente, programas adecuados de apoyo socioeconómico, condiciones propicias para el aprendizaje (métodos, infraestructura y recursos), mejoramiento del diseño y la administración curricular, procesos administrativos eficientes y orientación al estudiante con riesgos de desertar (Brenes, 2005).

Los antecedentes expuestos anteriormente coinciden con la información generada en el Informe Autoevaluación Carrera de Ciencias Geográficas con énfasis en Ordenamiento del Territorio (2009) y el informe de Implementación de la Guía Revista CAES Vol. 1, No. 1, Año 2010

ISSN-1659-4703 
Académica para Carrera de Ciencias Geográficas con énfasis en Ordenamiento del Territorio (2008). A partir de estos, la Escuela de Ciencias Geográficas de la Universidad Nacional propone una estrategia que permita disminuir la deserción, el tiempo de permanencia y dar seguimiento a los graduados en la carrera, la cual se comenta en las siguientes líneas.

\section{Estrategias de seguimiento para el éxito académico Asesoría académica}

Según el Plan Estratégico de la Escuela de Ciencias Geográficas (2007-2011) se formula como acción estratégica la ejecución de la Guía Académica desde de julio 2007. El objetivo se enfoca al cumplimiento de las metas de graduación, de permanencia y disminución de la deserción en los planes de estudio para las carreras de bachillerato y licenciatura en Ciencias Geográfica con énfasis en Ordenamiento del Territorio (CCG-UNA) y el diplomado en Cartografía y Diseño Digital (CDD-UNA).

La figura de guía académica se establece con la finalidad de acompañar al estudiante que ingresa a la carrera en su proceso formativo, disminuir la deserción, el rezago y facilitar su graduación. Los estudiantes de la carrera son apoyados de forma que obtengan los máximos beneficios educativos disponibles para ellos mediante la institución, ayudándoles a que se comprendan mejor ellos mismos, como entes sociales y estudiantes universitarios. Además como parte de su proceso de formación profesional instarles al uso de los recursos que ofrece la Universidad Nacional; obtener sus necesidades educativas así como sus aspiraciones y metas en general (Díaz y Solano, 2005).

La guía académica ofrecida es la orientación que reciben los estudiantes en su formación profesional integral durante el tiempo que permanezcan en la Escuela de Ciencias Geográficas. La naturaleza de esa labor debe ser congruente con la modalidad de la carrera y la forma en que se imparte la docencia tal y como lo establece la normativa institucional (Universidad Nacional, 2004).

Desde el año 2007 la CCG-UNA ofrece la asesoría académica a los estudiantes de primer ingreso, para ello se contó con la participación de tres docentes de la CCGUNA; durante el año 2008 se incorporó el grupo de primer ingreso, con lo cual se ofreció asesoría académica a estudiantes de I y II Nivel. En el año 2009 se incorporaron los estudiantes de primer ingreso, y los estudiantes de licenciatura Revista CAES Vol. 1, No. 1, Año 2010

ISSN-1659-4703 
mientras que la Subdirección asumió la asesoría del IV nivel. De esta forma quedó cubierta la totalidad de la población estudiantil matrícula en este plan de estudio. En la ECG-UNA se asignó Guía Académica a los estudiantes de I Ingreso del 2008, 2009 y 2010. Al año 2010 se tiene está cubierta la población estudiantil de esta unidad académica con su respectiva Guía Académica.

En la dinámica de trabajo de asesoría académica se han designado tres profesores por nivel, entre los cuales se distribuye de manera equitativa los estudiantes. De esta manera, los guías académicos trabajan de forma grupal e individual, en el nivel grupal coordinan con la Comisión Curricular y en el nivel individual se comunican y dan seguimiento a los estudiantes asignados. Las sesiones conjuntas han ayudado para la toma de decisiones en aspectos formales y problemas expuestos por los estudiantes. Asimismo, las funciones de los guías académicos se enfocan en asesorar a los estudiantes en la inducción y avance en el plan de estudio (requisitos y características de los cursos), inducción a la vida universitaria, la matrícula y remisión de estudiantes a los servicios de apoyo estudiantil y desarrollo académico. En cada ciclo lectivo los guías brindan información a la subdirección para mantener actualizado el expediente del estudiante. Para dar seguimiento a este nuevo proceso, se construyó una ficha para detallar información sobre el estudiante y observaciones del profesor del proceso de Guía Académica.

En cuanto a las carreras de CCG-UNA y CDD-UNA éstas han atendido el 80\% y el $65 \%$ de los estudiantes activos respectivamente. Todos los profesores (17) con carga superior al medio tiempo tienen asignada la asesoría académica, la forma de trabajo es ampliamente participativa y democrática. La capacitación a los guías académicos incluye el acompañamiento de orientación estudiantil e inducción al Sistema de Información Institucionalizado de la UNA (Banner). En los talleres realizados durante el año 2009 con estudiantes, éstos manifestaron que la guía académica es una fortaleza del plan de estudio.

El Programa de Éxito Académico ejecutado por Universidad Nacional, pretende construir un proceso articulado, sistemático de apoyo y acompañamiento institucional que contribuya al desarrollo integral y egreso exitoso de los estudiantes de la Universidad Nacional. Desde la Dirección de Docencia se apoya mediante tutorías de matemática, redacción, ortografía y técnicas de estudio. Además, la Vicerrectoría de Vida Estudiantil ofrece integración a la vida universitaria a través de los servicios de 
orientación, psicología, salud, bienestar estudiantil, actividades culturales y recreativas.

\section{Seguimiento de la deserción y rezago}

La permanencia del estudiante en las carreras de la ECG-UNA se sustenta en el compromiso de la Universidad y de la unidad académica por procurar el desarrollo integral de sus estudiantes. Para el análisis de la deserción y del rezago se dio seguimiento a las cohortes de los años de 1999 a 2007. De esta forma se compara el comportamiento de los indicadores en el plan trimestral (1999-2003) con el plan de ciclos (2004-2007). Los cuadros 2 y 3 muestran que en el plan trimestral la deserción oscilaba en el 50 \% y aún al año 2010 hay estudiantes de este plan rezagados. En el plan de ciclos se presenta una disminución de la deserción, excepto en el año 2007.

Cuadro 2. Estudiantes matriculados, desertores, graduados y rezagados en el plan trimestral para la Carrera de Ciencias Geográficas con énfasis en Ordenamiento del Territorio, 1999 a 2003

\begin{tabular}{|c|c|c|c|c|c|c|c|c|c|c|}
\hline \multirow{3}{*}{ Categoría } & \multicolumn{10}{|c|}{ Años } \\
\hline & \multicolumn{2}{|c|}{1999} & \multicolumn{2}{|c|}{2000} & \multicolumn{2}{|c|}{2001} & \multicolumn{2}{|c|}{2002} & \multicolumn{2}{|c|}{2003} \\
\hline & Abs & Rel & Abs & Rel & Abs & Rel & $A b s$ & Rel & Abs & Rel \\
\hline Matriculados & 15 & & 29 & & 38 & & 50 & & 48 & \\
\hline Deserción & 8 & 53,33 & 19 & 65,52 & 21 & 55,26 & 20 & 40 & 23 & 47,92 \\
\hline Graduados & 7 & 46,67 & 10 & 34,48 & 13 & 34,21 & 23 & 46 & 14 & 29,17 \\
\hline Rezagado & 0 & 0,00 & 0 & 0,00 & 4 & 10,53 & 7 & 14 & 11 & 22,92 \\
\hline Total & & 100,00 & & 100,00 & & 100,00 & & 100,00 & & 100,00 \\
\hline
\end{tabular}

Cuadro 3. Estudiantes matriculados, desertores, graduados y rezagados en el plan de ciclos para la Carrera de Ciencias Geográficas con énfasis en Ordenamiento del Territorio, 2004 a 2007

\begin{tabular}{|c|c|c|c|c|c|c|c|c|}
\hline \multirow{3}{*}{ Categoría } & \multicolumn{8}{|c|}{ Años } \\
\hline & \multicolumn{2}{|c|}{2004} & \multicolumn{2}{|c|}{2005} & \multicolumn{2}{|c|}{2006} & \multicolumn{2}{|c|}{2007} \\
\hline & Abs & Rel & Abs & Rel & $A b s$ & Rel & Abs & Rel \\
\hline Matriculados & 64 & & 46 & & 44 & & 54 & \\
\hline Deserción & 26 & 40,63 & 20 & 43,48 & 19 & 43,18 & 35 & 64,81 \\
\hline Graduados & 14 & 21,88 & 9 & 19,57 & 0 & 0,00 & 0 & 0 \\
\hline Rezagado & 24 & 37,50 & 17 & 36,96 & 0 & 0,00 & 0 & 0 \\
\hline Total & & 100 & & 100 & & 0,00 & & 0 \\
\hline
\end{tabular}


La ECG-UNA lleva un expediente de cada estudiante matriculado en la carrera, a partir esta información para los años 2006 y 2007 se caracterizó la población desertora. De un total de 54 estudiantes desertores se recuperó la información de 37 estudiantes. Entre las características sociodemográficas más destacadas se encuentra que la mayor deserción (54,05\%) son hombres, cerca del 89,19\% se encontraban solteros en el momento de la suspensión de sus estudios. El 51,35\% de los desertores provienen de colegios públicos y el 35, $83 \%$ proviene de familias compuestas por más de cuatro miembros. El 46,00\% de los desertores salieron de la UNA y el 32,00 \% realizaron traslado de carrera.

En el año 2007, con la finalidad de indagar las causas de la deserción se entrevistaron a seis de los desertores. Los estudiantes coincidieron en el preferencia por la Universidad Nacional y solamente una estudiante tenía como opción la CCGUNA. Al consultar si la administración de la Universidad pudo hacer algo para evitar la deserción de la carrera, una estudiante manifestó que si se le hubiera apoyado con la beca se hubiera quedado en la institución.

En coincidencia con las investigaciones revisadas, la mayor causa de deserción se relaciona con estudiantes que no tienen como preferencia la CCG-UNA. Dentro de las acciones que reorientan la gestión curricular en los años siguientes se ha procedido a ser más selectivos con los estudiantes que solicitan admisión a la CCG-UNA.

En el año 2009 se procedió a identificar los estudiantes con retraso en el plan de estudio y actualmente se toman acciones para identificar las causas y las posibles soluciones con la finalidad que éstos puedan culminar el plan de estudio.

A nivel institucional, las diferentes instancias de la UNA han realizado acciones para prevenir la deserción: se tiene información actualizada de los estudiantes que hacen deserción, de los que realizan suspensión temporal, los estudiantes egresados no titulados, aquellos que hacen reintegro a carrera. De esta forma la asesoría académica facilita la detección temprana de posibles desertores, también se brinda apoyo tutorial en Matemática Básica y se ofrecen actividades de orientación vocacional. 


\section{VII.Seguimiento de graduados}

Los estudios de seguimiento de graduados surgen como una necesidad de las instituciones de educación superior de ajustar sus programas y planes de estudio a las cambiantes demandas del mundo de trabajo y de la economía globalizada (Salas, 2006). En el campo de la Geografía, países como México iniciaron desde el año 1992 el seguimiento de graduados (Urióstegui, 1995). Desde esta perspectiva la actualización de los planes de estudio debe de tomar en consideración la opinión de los diferentes actores involucrados, entre éstos, los profesores, los graduados, los estudiantes y los empleadores.

En el año 2005, la ECG-UNA inició un proceso de autoevaluación que incluyó el seguimiento a graduados, desde este momento se convirtió en una actividad permanente que monitorea algunos indicadores como tasa de colocación, jornada laboral, tiempo para conseguir el primer empleo, tipo de empleo, relación entre las tareas realizadas y la formación recibida, descripción detalladas de la tareas realizadas, tipo de institución donde laboran, satisfacción de la formación recibida, satisfacción del trabajo desempeñado, empresas donde laboran y demanda de la carrera.

La tasa de colocación del graduado en Ciencias Geográficas, entre los años 2005 y 2008 fue de $87,00 \%$ y el $89,00 \%$ respectivamente. La entidad para la cual laboran los graduados de la carrera presentó un aumento de los que fueron contratados en el sector público (70,30\%), especialmente en las municipalidades. Las empresas que mayormente contrataron graduados en Geografía para el periodo 2002 a 2008 están vinculadas al sector servicios cartográficos, ambientales, educación, transporte, telecomunicaciones, entre otros.

Entre el $88,00 \%$ y $100,00 \%$ del total de graduados (2002-2008) que se encuentran laborando mencionan que el grado de relación entre la carrera estudiada y las funciones que desempeñan está completamente relacionado y relacionado con la formación recibida. Para 2008, sobresalen las funciones que realizan Sistemas de Información Geográfica con 90,24 \%, en Cartografía ejerce el 82,92\%; el 73,17\% cumple funciones de Teledetección, Fotogrametría y Sistemas de Posicionamiento Global (GPS), un 34,15\% elaboran Estudios, Auditorias y Evaluación de Impacto Ambiental, entre otras funciones de las diez y seis identificadas. 
Entre el $90,17 \%$ y $89,80 \%$ de los que laboran lo hacen a tiempo completo o más de 40 horas semanales. Para el año 2008, el 54,83\% de los encuestados obtuvieron su primer empleo en el transcurso de la carrera y de estos el $57 \%$ lo obtuvieron en menos de 6 meses y un 28,57 \% fueron contratados entre los 6 y 1 año. El 75,61\% manifiesta que su primer empleo fue en el mismo ámbito de la formación académica que recibió en la Universidad.

En cuanto a la opinión en la demanda de la carrera en el mercado laboral ésta varió en el período analizado, en el 2005 únicamente el 13,80\% de los graduados la consideran como muy buena y buena y en el 2008 aumentó a un 34,15\%, en tanto el $53,66 \%$ señalaron que es regular. La satisfacción de las tareas realizadas, como un indicador de calidad, presentó una mejoría positiva, en el 2005 el 60,00\% manifestaron estar muy satisfechos y satisfechos con el trabajo realizado, para el 2008 la satisfacción aumentó a 95,00\%.

Los resultados del seguimiento de graduados han sido utilizados en la gestión administrativa y curricular del plan de estudio. Actualmente se cuenta con una bolsa de empleo que posibilita la colocación, cada año se ofrece un curso de formación continua a los graduados y la actualización del plan de estudio toma en cuenta las opiniones de los diferentes grupos involucrados (Araya, 2010:146-148).

\section{Conclusiones}

A nivel del país, los datos sobre la deserción y la prolongación de los estudios universitarios es un tema que repercute a los actores involucrados, especialmente las aspiraciones de los propios desertores. La identificación de los factores de deserción, contribuyen a lo interno de la unidad a referencia aquellas situaciones que de alguna manera se pueden solventar para evitar el fracaso académico de los estudiantes.

Las estrategias implementadas por la ECG como la Guía Académica y el seguimiento a graduados, permiten identificar elementos de planificación que ayudan a disminuir los porcentajes de la población que deserta. La generación de líneas de trabajos y las demandas de la sociedad, contribuyen a la atracción de una población satisfecha con la elección y a finalizar exitosamente sus estudios.

El contar con indicadores precisos como reprobación de cursos, cursos con alta repitencia, y aspectos puntuales como evaluaciones individuales de desempeño docente, contribuyen a la toma de decisiones informadas, y el fortalecimiento de 
estrategias que involucran a los sectores del proceso educativo, administración, docentes y estudiantes.

La graduación exitosa de una cohorte, va a depender del esfuerzo y la motivación con que se trabaje esta población, que sin duda alguna se verá influida por situaciones personales particulares, pero que necesariamente los procesos de seguimiento y evaluación deben colocarse como ejes centrales en la formación universitaria.

\section{Bibliografía}

Abarca, A. y Sánchez, A. (2005). La deserción estudiantil en la educación superior: el caso de la Universidad de Costa Rica. Revista Electrónica Actualidades Investigativas en Educación. Volumen 5, Número Especial, Año 2005, ISSN 1409-4703. Extraído http://revista.inie.ucr.ac.cr/articulos/extracea/archivos/desercion.pdf. Consultado el día 11 de junio de 2007.

Araya, I, Quirós, L., Morera, C., Alfaro, D., Hernando, L. y Rodríguez, S. (2009). Informe Autoevaluación Carrera de Ciencias Geográficas con énfasis en Ordenamiento del Territorio. Universidad Nacional. Costa Rica.

Araya, I. (2010). Inserción laboral y líneas de trabajo del geógrafo de la Universidad Nacional. En Revista Geográfica de América Central, № 44. pp 143-178.

Araya I, Quirós L (2008). Implementación de la Guía Académica para la carrera de Ciencias Geográficas con énfasis en Ordenamiento del Territorio. Universidad Nacional. Costa Rica (Manuscrito no publicado).

Araya I, Quirós L (2008). Implementación de la Guía Académica para las carreras de Ciencias Geográficas con énfasis en Ordenamiento del Territorio Cartografía y diseño digital, 2007-2009. . Universidad Nacional. Costa Rica (Manuscrito no publicado).

Brenes, M. (2005). Deserción y Repitencia en la Educación Superior Universitaria de Costa Rica. Consejo Nacional de Rectores, Oficina de Planificación de la Educación Superior. 
Brenes, M. (2005). Aspectos relacionados con el rendimiento académico de los estudiantes que ingresaron a las instituciones de educación superior universitaria estatal en 1996. Consejo Nacional de Rectores, Oficina de Planificación de la Educación Superior.

Brenes, M. (2003). Los géneros en la educación superior universitaria en Costa Rica. Consejo Nacional de Rectores, Oficina de Planificación de la Educación Superior.

Cabrera, L; Bethencourt, J, González, A y Álvarez P. (2006). Un estudio transversal retrospectivo sobre prolongación y abandono de estudios universitarios. RELIEVE, v. 12, n. 1. http://www.uv.es/RELIEVE/v12n1/RELIEVEv12n1_1.htm. Consultado en 24 de noviembre de 2009.

CONARE-OPES (2005). Plan Nacional de la Educación Superior Universitaria Estatal 2006-2010. San José Costa Rica.

Díaz, E y Solano, A (25 de octubre de 2007). Proceso de implementación del Guía Académico para estudiantes de la Carrera Bachillerato y Licenciatura en la Enseñanza de la Matemática. Ponencia presentada en la III Jornada de Intercambio de Experiencias Innovadoras en la Educación Universitaria "Vida Estudiantil y su impacto en una formación universitaria de excelencia". Universidad Estatal a Distancia.

Escuela de Ciencias Geográficas (2004). Plan de Estudios de la Carrera de Ciencias Geográficas con énfasis en Ordenamiento del Territorio. Universidad Nacional. Costa Rica.

González, R. (2004). La situación laboral y otras características de los graduados del 2001 de las Universidades estatales. Consejo Nacional de Rectores, Oficina de Planificación de la Educación Superior. CONARE, OPES publicaciones San José, C.R.

López, M; Quiceno, M (2003). La deserción estudiantil del programa de administración del medio ambiente 1996-2001. Colombia. Extraído del sitio Revista CAES Vol. 1, No. 1, Año 2010 ISSN-1659-4703 
web de Universidad Tecnológica de Pereira el 16 de julio de 2007. http://www.utp.edu.co/facultad/ambiental/archivos/deser.est.pdf.

Morera, C., Alfaro, D., Araya, I. y Quirós, L. (2006). Plan Estratégico Escuela de Ciencias Geográficas 2007-2012. Universidad Nacional. Heredia. Costa Rica. 17 $\mathrm{p}$ (mimeografiado).

Navarro, G. (2007). Impacto del proceso de acreditación de carreras en el mejoramiento de la gestión académica. Revista Calidad en la Educación № 26, julio 2007 pp. 245-288.

Quirós L (2009). Geography Education in Costa Rica In Muñiz, O y Boehm R (editors) Geography Education pan American Perspectives. Ed Grosvenor Center for Geography Education. United Satate.pp 205-230.

Sandoval, P., Núñez, E., Solís, M., Rodríguez., O; Guevara., G; Calderón., W (2008)

Causas de la deserción en los estudiantes de primer ingreso (periodo 2003 al 2006) de la Universidad Nacional. Vicerrectoría de Vida Estudiantil, Instituto de estudios en Población, Área de Planificación Económica. Universidad Nacional.

Sandoval, P., Núñez, E., Solís, M., Rodríguez., O; Guevara., G; Calderón., W (2008) Causas de rezago de los estudiantes regulares de la Universidad Nacional. Vicerrectoría de Vida Estudiantil, Instituto de estudios en Población, Área de Planificación Económica. Universidad Nacional.

Salas, F. (2006). Los estudios de seguimiento a la población graduada como herramienta para el cambio y la innovación en el currículo universitario. Revista Educación, Año 30, № 002, pp 63-81.

Universidad Nacional (2004) Lineamientos sobre la guía académica En UNA GACETA, Nº 24 del 10 de diciembre del 2004.

Urióstegui, A. (1995) Seguimiento de egresados para la licenciatura en Geografía. Generación 1992, en la ciudad de Toluca. Revista Papeles de Población, № 6-7 (noviembre-marzo), pp 85-98.

\footnotetext{
' http://www.sinaes.ac.cr

Revista CAES Vol. 1, No. 1, Año 2010
}

ISSN-1659-4703 